\title{
Evidencias de validez y fiabilidad de la versión en español del Children's Depression Inventory-Short en población peruana
}

\author{
José Ventura-León, Tomás Caycho-Rodríguez, Shirley Tocto-Muñoz, Maryuri Torres-Maldonado y \\ Karen Curahua-Guillén
}

Universidad Privada del Norte, Lima, Perú

Evidence of validity and reliability of the Spanish version of the Children's Depression Inventory-Short in Peruvian population

\begin{abstract}
The objective of this study was to examine the validity evidence of the Children's Depression Inventory-Short (CDI-S; Kovacs, 1992), in Peruvian school children. A total of 1059 children between 7 and 12 years old participated $($ Mean $=9.73$, $S D=1.23$ ). The results revealed the existence of two factors related to strong loads in the exploratory factor analysis, which were corroborated by confirmatory factor analysis with good goodness-of-fit indices (CFI $\geq .97$, RMSEA $\leq .04)$. The reliability of the CDI-S, calculated with the internal consistency method, was good in both factors $(\omega>.80)$. We conclude that the CDI-S is a valid and reliable measure in Peruvian children and should be interpreted as a test with two factors (Dysphoria and Negative self-esteem), which is consistent with previous empirical and theoretical studies.
\end{abstract}

Keywords: Validation; reliability; depression; children.

Resumen: El presente estudio tuvo como objetivo examinar evidencias de validez y fiabilidad de la versión en español del Children's Depression Inventory-Short (CDI-S, Kovacs, 1992), en población peruana. Participaron 1059 niños y niñas entre 7 y 12 años $($ Media $=9.73, D T=1.23)$. Los resultados revelaron la existencia de dos factores relacionados con cargas fuertes en el análisis factorial exploratorio, los cuales fueron corroborados mediante el análisis factorial confirmatorio con buenos índices de bondad de ajuste (CFI $\geq .97$, RMSEA $\leq .04$ ). La fiabilidad del CDI-S, calculada con el método de consistencia interna, fue buena en los dos factores $(\omega>.80)$. Se concluye que el CDI-S es una medida valida y fiable en niños peruanos y debe ser interpretada como una prueba con dos factores (Disforia y Autoestima negativa), lo cual es coherente con estudios empíricos y teóricos previos.

Palabras clave: Validación; fiabilidad; depresión; niños.

\section{Introducción}

Se estima que la depresión afecta a más de 300 millones de personas, lo cual representa el $4.4 \%$ de la población mundial (Camuñas, Mavrou, y Miguel-Tobal, 2019). Diferentes estudios señalan que esta cifra se in-

Recibido: 17 de diciembre 2019; aceptado: 24 de abril 2020 Correspondencia: Dr. José Ventura-León, Facultad de Salud, Universidad Privada del Norte, Av. Alfredo Mendiola, 6062 Los Olivos, Perú. Correo-e: jose.ventura@upn.pe crementa año tras año y que en el 2020 la depresión será el segundo padecimiento mental y físico en el mundo y que causará una elevada morbilidad (Jaureguizar, Bernaras, y Garaigordobil, 2017). Asimismo, este padecimiento aparece en cualquier etapa de desarrollo, incluida la infancia (Costello, Copeland, y Angold, 2011), aunque su presencia en esta población ha resultado cuestionada y controvertida (Alcázar et al., 2002).

Diversos estudios han informado sobre la presencia de síntomas depresivos en niños a partir de los 6 u 8 años (Bernaras-Iturrioz, Jauregizar-Albonigamayor, So- 
roa-Udabe, Ibabe-Erostarbe, y De las Cueva-Hevia, 2013). La prevalencia en niños menores de 13 años es del $2.8 \%$, encontrándose la mayor concentración de casos entre los 6 y los 12 años (Jaureguizar et al., 2017), con una prevalencia total de entre el $4 \%$ y el $25 \%$ (Bernaras-Iturrioz et al., 2013; Demir, Karacetin, Demir, y Uysal, 2011). Estas cifras se elevan en niños con enfermedades médicas como migraña, epilepsia, cardiopatía congénita y cáncer, entre otros, donde las cifras se sitúan entre el 11 y el $29 \%$ (Allgaier et al., 2012). En Hispanoamérica, entre el $35 \%$ y el $43 \%$, aproximadamente, de los niños presentan síntomas depresivos (Del Barrio, 1997). En Perú, se ha constatado que el $3.5 \%$ de los niños de entre 6 y 10 años experimenta sintomatología depresiva (Instituto Nacional de Salud Mental Honorio Delgado-Hideyo Noguchi, 2018). Estos datos sugieren que la depresión infantil constituye un serio problema de salud pública que requiere llevar a cabo intervenciones efectivas (Sun y Wang, 2015). Sin embargo, estas intervenciones no podrán ser realizadas sino se cuenta con un instrumento de medida adaptado a estas edades.

En el ámbito escolar, la depresión se encuentra asociada a las bajas calificaciones (Borges y Angeli Dos Santos, 2016) y a la vulnerabilidad a sufrir bullying (Lara-Ros, Rodríguez-Jiménez, Martínez-González, y Piqueras, 2017). La depresión influye en las conductas de rebeldía o de protesta que pueden manifestarse en el entorno escolar (Del Barrio, 1997). Asimismo, en el ámbito familiar la depresión se ha relacionado con los estilos de crianza (McLeod, Weisz y Wood, 2007) y el funcionamiento familiar, encontrándose una relación inversa entre la depresión y la comunicación, el cumplimiento de las tareas y la implicación afectiva (Martinez y Rosselló, 2015). En el ámbito social se ha demostrado que los niños testigos de violencia callejera experimentan mayores tasas de depresión (Quintana y Maldonado, 2016).

Considerando que la depresión infantil afecta negativamente a la salud y tiende a persistir en la adolescencia (Delmas et al., 2011), su identificación en edades tempranas es importante para reducir sus efectos negativos y mejorar la calidad de vida de los menores (De la Vega et al., 2016). De hecho, se estima que la tasa diagnóstica de la depresión en la infancia y la adolescencia ronda solo el 12.5 \% (Allgaier et al., 2012). En ese sentido, resulta necesario contar con cuestionarios de autoinforme para la detección de síntomas depresivos en edades tempranas (Stevanovic, 2012; Sun y Wang, 2015); estos cuestionarios deberían ser pruebas breves que puedan ofrecer resultados fiables (Ivarsson, Svalander, y Litlere, 2006).
Una de las pruebas de autoinforme más utilizadas para la evaluación de la depresión infantil es el Children's Depression Inventory (CDI) de 27 ítems (Kovacs, 1992), el cual ha sido traducido a diferentes idiomas (Allgaier et al., 2012) y cuenta con una versión corta de 10 ítems, denominada Children's Depression Inventory Short Version (CDI-S), resultado de la eliminación de los ítems que presentaban bajas correlaciones entre ellos (De la Vega et al., 2016). El CDI-S podría ser un instrumento de tamizaje para la detección temprana de síntomas depresivos en el entorno escolar.

Hasta la fecha son pocos los estudios que han revisado las propiedades psicométricas del CDI-S en población infantil. Se ha informado de su fiabilidad con muestras de diferentes países entre los que se incluyen España $(\alpha=.80$; Del Barrio, Capilla, Olmedo, y Colodrón, 2002), Irlanda ( $\alpha=.79$; Meehan, Houghton, Cowley, Houghton, y Kelleher, 2008), Serbia ( $\alpha=.74$; Stevanovic, 2012), Alemania ( $\alpha=.70$; Allgaier et al., 2012), Chile $(\alpha=.84$; Caqueo-Urízar, Urzúa y de Munter, 2014) y Colombia ( $\alpha=.67$ a .77; Yánez et al., 2017). Asimismo, una investigación de meta-análisis que incluye 22 estudios sobre el CDI-S informó que el coeficiente $\alpha$ se situaba en torno al .77 (Sun y Wang, 2015).

Por otro lado, también se ha examinado la estructura interna del CDI-S. En un estudio realizado en España se informó de una estructura de tres factores: autoestima, anhedonia y desesperanza (Del Barrio et al., 2002). En el estudio de Yánez et al. (2017) realizado en Colombia se aislaron dos factores que fueron asociados a la depresión y la ansiedad, respectivamente. En un estudio con 1262 niños de escuelas suecas se encontró que la variación en las puntuaciones se explicaba por la presencia de un factor general (Ahlen y Ghaderi, 2017). La estructura unifactorial del CDI-S fue validada a partir de un análisis factorial confirmatorio (AFC), el cual concluyó la presencia de un solo factor con buenas bondades de ajuste (Stevanovic, 2012). Estos estudios han demostrado que la presencia de diferentes estructuras factoriales del CDI-S no afecta a su capacidad diagnóstica (Allgaier et al., 2012; del Barrio et al., 2002; Kovacs, 1992). Pese a ello, resulta necesario verificar su estructura en un nuevo contexto, debido a las variaciones que presenta en diferentes países.

La presente investigación tiene los siguientes objetivos: (a) evaluar la estructura interna del CDI-S mediante análisis factorial exploratorio (AFE) y AFC para determinar la estructura factorial del CDI con una muestra de niños residentes en Perú; (b) examinar la fiabilidad mediante el método de consistencia interna y (c) examinar la relación del CDI-S con su versión de 27 ítems. 


\section{Método}

\section{Participantes}

Se aplicó el CDI-S (Kovacs, 1992) en dos momentos del 2019. En primer lugar, se contó con la participación de 1059 niños y niñas de siete instituciones educativas privadas y públicas de las ciudades de Lima Metropolitana y Callao, instituciones que fueron seleccionadas por muestreo intencional, bajo el criterio de accesibilidad. El 539 de los participantes tenía sexo biológico femenino $(50.9 \%)$ mientras que 520 tenía sexo masculino (49.1 $\%)$. La edad de estos participantes estaba comprendida entre los 7 y los 12 años $(M=9.73 ; D T=1.23)$. Los 1059 participantes fueron distribuidos mediante un método computacional aleatorio a uno de los dos momentos del análisis factorial (véase la Tabla 1). En segundo lugar, 10 semanas después fue aplicada la versión de 27 ítems del CDI y el CDI-S (Kovacs, 1992) en una nueva institución educativa privada a 99 participantes, de los cuales el $46.4 \%$ tenía sexo biológico femenino. La edad de esta segunda muestra estuvo comprendida entre los 8 y los 13 años $($ Media $=10.14 ; D T=1.20)$.

\section{Instrumentos}

Children's Depression Inventory - Short Version (CDI-S, Kovacs, 1992). En la versión española de Del Barrio et al., (2002), que evalúa síntomas depresivos durante las 2 semanas últimas. Está compuesto por 10 ítems, con una escala tipo Likert de 3 puntos $(0=$ ausencia del síntoma, $1=$ presencia baja y $2=$ presencia de intensidad alta). Los puntajes más altos indican síntomas depresivos más intensos. Está conformado por tres factores: (a) autoestima $(10,4,7,8)$ que consiste en una valoración negativa de sí mismo; (b) anhedonia $(5,1,6)$ que involucra incapacidad para experimentar placer expresado en ganas de llorar, tristeza y preocupación recurrente; y (c) desesperanza $(2,3,9)$ relacionado con el desánimo, evaluación negativa de las actividades y anhelo de conseguir más amigos. Cabe señalar que en los ítems 2, 4, 5, 6 y 10 deben invertirse las puntuaciones. El CDI-S está diseñado para niños de 7 años en adelante. De acuerdo con Del Barrio et al. (2002), esta versión breve presenta una alta correlación $(r=.89)$ con la versión completa de 27 ítems (CDI-S; Kovacs, 1992). En este estudio, la validez del instrumento se valoró mediante AFE a través del método de componentes principales y rotación varimax, demostrándose que los tres factores explican el $50 \%$ de la varianza. Respecto a la fiabilidad, en la presente muestra fue aceptable ( $\alpha$ de Cronbach $=.75$ ).
Tabla 1. Características sociodemográficas de la muestra total y las submuestras

\begin{tabular}{|c|c|c|c|}
\hline Sexo & $\begin{array}{c}\text { Total } \\
n=1059\end{array}$ & $\begin{array}{c}\text { AFE } \\
n=250\end{array}$ & $\begin{array}{c}\text { AFC } \\
n=809\end{array}$ \\
\hline Sexo femenino $[n(\%)]$ & $539(50.9)$ & $134(53.6)$ & $405(50.1)$ \\
\hline Sexo masculino $[n(\%)]$ & $520(49.1)$ & $116(46.4)$ & $404(49.9)$ \\
\hline \multicolumn{4}{|l|}{ Edades $[n(\%)]$} \\
\hline 7 & $4(0.4)$ & - & $4(0.5)$ \\
\hline 8 & $188(17.8)$ & $42(16.8)$ & $146(18.0)$ \\
\hline 9 & $310(29.3)$ & $73(29.2)$ & $237(29.3)$ \\
\hline 10 & $225(21.2)$ & $61(24.4)$ & $164(20.3)$ \\
\hline 11 & $244(23.0)$ & $49(19.6)$ & $195(24.1)$ \\
\hline 12 & $85(8.0)$ & $25(10.0)$ & $60(7.4)$ \\
\hline \multicolumn{4}{|l|}{ Instituciones educativas $[n(\%)]$} \\
\hline 1 & $183(17.3)$ & $42(16.8)$ & $141(17.4)$ \\
\hline 2 & $230(21.7)$ & $47(18.8)$ & $183(22.6)$ \\
\hline 3 & $59(5.6)$ & $18(7.2)$ & $41(5.1)$ \\
\hline 4 & $115(10.9)$ & $30(12.0)$ & $85(10.5)$ \\
\hline 5 & $190(17.9)$ & $40(16.0)$ & $150(18.5)$ \\
\hline 6 & $73(6.9)$ & $20(8.0)$ & $53(6.6)$ \\
\hline 7 & $209(19.7)$ & $53(21.2)$ & $156(19.3)$ \\
\hline \multicolumn{4}{|l|}{ Grado escolar $[n(\%)]$} \\
\hline $3^{\circ}\left(3^{\circ}\right.$ de Primaria en España $)$ & $311(29.4)$ & $74(29.6)$ & $237(29.3)$ \\
\hline $4^{\circ}\left(4^{\circ}\right.$ de Primaria en España) & $312(29.5)$ & $68(27.2)$ & $244(30.2)$ \\
\hline $5^{\circ}\left(5^{\circ}\right.$ de Primaria en España $)$ & $163(15.4)$ & $51(20.4)$ & $112(13.8)$ \\
\hline $6^{\circ}\left(6^{\circ}\right.$ de Primaria en España $)$ & $273(25.8)$ & $57(22.8)$ & $216(26.7)$ \\
\hline
\end{tabular}

\section{Procedimiento}

El procedimiento para la adaptación del test siguió las directrices del International Test Commission (ITC, 2018). De ese modo, se hizo uso de la entrevista semiestructurada con una muestra de diez niños, con la finalidad de comprobar la comprensión de los ítems, proceso que es necesario para disminuir la aparición de sesgo en los ítems por motivos lingüísticos y garantizar así la equivalencia semántica. A raíz de la entrevista, se efectuó un ligero cambio en el ítem 7, añadiendo a la expresión «tengo buen aspecto» el término «físico».

Una vez se contaba con una versión final del CDI-S, ésta fue administrada de modo colectivo y presencial en siete instituciones educativas en horario escolar. Posteriormente, en una institución educativa diferente, se administró la versión corta y extensa del CDI. 
En todos los centros educativos, los investigadores se coordinaron con las autoridades de las instituciones educativas y los padres firmaron un consentimiento informado en el cual se explicó el propósito del estudio, el procedimiento, los riesgos, los beneficios, la confidencialidad y el uso futuro de la información recogida. Los cuestionarios fueron aplicados por parte de tres asistentes de investigación por aula, quienes estaban capacitados para responder dudas o inquietudes de los estudiantes y procuraron leer las instrucciones de la prueba como forma de estandarizar el proceso de administración del cuestionario.

\section{Análisis estadístico}

Se utilizaron dos programas: (a) FACTOR versión 10.8 (Lorenzo-Seva y Ferrando, 2006) y (b) Rstudio con la librería lavaan (Rosseel, 2012). Respecto al análisis de los datos, este se realizó en cinco etapas:

En primer lugar, se calcularon los estadísticos descriptivos de los ítems (media, desviación estándar, asimetría, curtosis). En segundo lugar, se efectuó un análisis factorial exploratorio (AFE) con el fin de examinar cómo se distribuyen los ítems sin restricción alguna. Se estimaron las correlaciones policóricas a partir de una muestra de 250 participantes (Freiberg Hoffmann, Stover, de la Iglesia, y Fernández-Liporace, 2013). Se examinó la factorización a través del coeficiente KMO y la prueba de esfericidad de Bartlett; mientras que para la determinación del número de factores se utilizó el método de análisis paralelo (Timmerman y Lorenzo-Seva, 2011). El método de estimación de factores fue mínimo cuadrado no ponderados, debido a la distribución no normal de los datos (Osborne, Costello y Kellow, 2008) calculados mediante la asimetría y curtosis y el método de rotación fue Promin por tratarse de factores correlacionados.

En tercer lugar, se verificó la estructura factorial obtenida en el AFE por medio del análisis factorial confirmatorio (AFC). Siguiendo las recomendaciones de Mueller y Hancock (2008), se reportaron los siguientes índices de bondad de ajuste: $\chi 2 / \mathrm{gl}(<2)$, RMSEA $\leq .06$, $\mathrm{SRMR} \leq .08, \mathrm{CFI} \geq .95$ (Hu y Bentler, 1999). Además, se modeló una estructura bifactor (un factor general con dos factores específicos); a pesar de que no existió una fuerte relación entre los factores, se probó con la finalidad de contrastar lo previamente encontrado (Ahlen y Ghaderi, 2017). En ese sentido, se estimaron algunas medidas de ajuste, como el ECV (Explained Common Variance General), que representa la proporción de varianza común que puede ser atribuida al factor general $\omega_{h}$ (omega jerárquico) y $\omega_{h s}$ (omega jerárquico para la subescala) que, respectivamente, indican la proporción de varianza explicada por el factor general y el factor específico (Reise, Scheines, Widaman, y Haviland, 2013); asimismo, valores del $\omega_{h s}$ entre .20 y .30 indican una moderada proporción de la varianza (Smits, Timmerman, Barelds, y Meijer, 2014).

En cuartó lugar, se estimó la fiabilidad mediante el coeficiente omega $(\omega)$ al utilizarse un enfoque factorial (Ventura-León y Caycho-Rodríguez, 2017).

En quinto lugar, se realizaron correlaciones de Pearson para evidenciar la cantidad de correlación que existe entre la versión corta y extensa del CDI, conociendo que existía una correlación gran $(\mathrm{r}=.89)$ entre ambas medidas (Del Barrio et al., 2002). Asimismo, para la interpretación de la magnitud de la correlación se utilizaron las recomendaciones de Cohen (1988) donde: $r=.10$ a .30 , es pequeño; $r=.31$ a .50 , moderado y $r \geq .51$, grande.

\section{Resultados}

\section{Análisis descriptivos de los ítems}

En la Tabla 2, se presentan los estadísticos descriptivos de los ítems (media aritmética, desviación estándar, asimetría y curtosis) para los dos momentos del análisis factorial. Se observa que el ítem 7 presenta la media más alta en ambas condiciones y la más baja la ostenta el ítem 1. Respecto a la asimetría y la curtosis, los ítems 1 , 3,4 y 10 sobrepasan el valor $+/-1.5$; esto sugiere el incumplimiento del supuesto de normalidad multivariada.

\section{Evidencia basada en la estructura interna}

En la Tabla 3, se presentan los resultados del AFE considerándose preliminarmente las medidas de adecuación muestral que verificaron que era posible realizar el análisis factorial $(\mathrm{KMO}=.72$, Bartlett $=p<0.001)$. Aquellos obtenidos mediante el análisis paralelo sugieren la presencia de dos factores que explican el 59.03\% de la varianza del constructo. Los mismos que son coherentes con la versión original (Kovacs, 1992) y son síntomas principales de la depresión infantil desde hace mucho tiempo (Weinberg, Rutman, Sullivan, Penick, y Dietz, 1973). De esta manera, el factor 1 recibe el nombre de Disforia y está conformado por los ítems 2, 5, 6 y 7 , que expresan cambios en el estado de ánimo, incapacidad, sentimiento de fealdad y facilidad para el llanto; mientras que el factor 2 es denominado Autoestima negativa y agrupa a los ítems $1,3,4,8,9$ y 10 , que contienen expresiones de tristeza, soledad, autorechazo, indefensión y pesimismo. Las cargas fueron $\geq .40$ y la correlación entre los factores fue moderada $(\phi=.39)$. La 
Tabla 2. Análisis preliminar de los ítems del CDI

\begin{tabular}{ccccccc}
\hline \multirow{2}{*}{ Ítems } & \multicolumn{3}{c}{ AFE } & $n=250$ & & \multicolumn{3}{c}{ AFC } \\
& \multicolumn{1}{c}{$M(D T)$} & $\mathrm{g} 1$ & $\mathrm{~g} 2$ & $M(D T)$ & $\mathrm{g} 1$ & $\mathrm{~g} 2$ \\
\cline { 2 - 7 } & $0.14(0.44)$ & 3.18 & 9.44 & $0.13(0.44)$ & 3.86 & 16.66 \\
$2^{*}$ & $0.71(0.74)$ & 0.52 & -1.03 & $0.74(0.75)$ & 0.47 & -1.08 \\
3 & $0.26(0.56)$ & 2.04 & 3.07 & $0.19(0.47)$ & 2.43 & 5.21 \\
$4^{*}$ & $0.16(0.45)$ & 2.91 & 7.70 & $0.16(0.43)$ & 2.82 & 7.44 \\
$5^{*}$ & $0.60(0.90)$ & 0.88 & -1.18 & $0.57(0.89)$ & 0.94 & -1.06 \\
$6^{*}$ & $0.73(0.89)$ & 0.55 & -1.50 & $0.71(0.87)$ & 0.59 & -1.43 \\
7 & $1.34(0.76)$ & -0.64 & -0.97 & $1.28(0.75)$ & -0.51 & -1.06 \\
8 & $0.38(0.64)$ & 1.48 & 0.94 & $0.31(0.57)$ & 1.73 & 1.94 \\
9 & $0.42(0.59)$ & 1.08 & 0.17 & $0.41(0.55)$ & 0.92 & -0.20 \\
$10^{*}$ & $0.21(0.52)$ & 2.44 & 4.95 & $0.21(0.48)$ & 2.29 & 4.54 \\
\hline
\end{tabular}

Nota. $M=$ Media; $D T=$ Desviación típica; g1 = Asimetría; g2 = Curtosis; *Ítem inverso.

fiabilidad en ambos factores fue $\omega>.80$, que es considerado bueno.

Tabla 3. Análisis Factorial Exploratorio del CDI $(n=250)$

\begin{tabular}{|c|c|c|c|}
\hline Ítems & $\mathrm{F} 1$ & $\mathrm{~F} 2$ & $h^{2}$ \\
\hline 2 & .82 & -.05 & .64 \\
\hline 5 & .90 & .05 & .85 \\
\hline 6 & .43 & .07 & .21 \\
\hline 7 & .86 & -.09 & .68 \\
\hline 1 & -.05 & .71 & .48 \\
\hline 3 & .11 & .56 & .38 \\
\hline 4 & .01 & .84 & .72 \\
\hline 8 & -.07 & .71 & .47 \\
\hline 9 & -.02 & .42 & .17 \\
\hline 10 & -.01 & .69 & .47 \\
\hline Autovalor & 3.90 & 2.00 & \\
\hline$\%$ de varianza & 39.05 & 19.98 & 59.03 \\
\hline$\phi$ & & & \\
\hline$\omega$ & .85 & .82 & \\
\hline
\end{tabular}

Nota. F1 = Disforia $;$ F2 = Autoestima negativa $h^{2}=$ Comunalidad antes de la rotación; $\omega=$ Coeficiente Omega; $\phi=$ Correlación inter-factorial

En la Tabla 4, se evidencia el AFC que se efectuó con la finalidad de verificar y comparar la estructura interna del CDI-S. Para ello, se compararon cuatro modelos: a) Modelo 1, con un solo factor (Stevanovic, 2012); b) Modelo 2, con tres factores relacionados entre sí, versión original (Del Barrio et al., 2002); c) Modelo 3, dos factores oblicuos basados en los resultados previos del AFE; y d) Modelo 4, modelo bifactor, un factor general y dos factores específicos ortogonales (Ahlen y Ghaderi, 2017). De acuerdo con los resultados obtenidos, los modelos 3 y 4 compiten respecto a las mejores medidas de bondad de ajuste. No obstante, el $\chi 2 / \mathrm{gl}$ es inferior en el modelo 3. Asimismo, mediante un análisis de las medidas auxiliares para modelos bifactor, como el ECV, $\omega_{h} \mathrm{y}$ $\omega_{h s}$, se descarta la idea de bifactorialidad porque la varianza común explicada es débil $(\mathrm{ECV}=.35)$. Asimismo, una moderada y alta cantidad de varianza es explicada por los dos factores específicos $\left(\omega_{F 1}=.76\right.$ y $\left.\omega_{F 2}=.35\right)$. Es así que se prefiere el modelo 3. Por otro lado, el modelo tridimensional (Del Barrio et al., 2002) no logra converger debido a la presencia de una matriz positiva no definida, que ocurre por la alta relación inter-factorial $(\phi>.95)$.

\section{Fiabilidad}

En la Figura 1, se presentan las cargas factoriales del modelo 3 , que son fuertes $(\lambda>.50)$, lo que evidencia que el coeficiente $\omega$ para Disforia fue .89 y para Autoestima negativa, .81 .

\section{Evidencia de relación con otras variables}

Una vez comprobada la bidimensionalidad del CDI-S se efectuaron correlaciones de Pearson con la finalidad de examinar su relación con la versión de 27 ítems del CDI observándose que entre la versión breve y extensa 
Tabla 4. Índices de ajuste estadístico de los 4 modelos del CDI-S $(\mathrm{n}=809)$

\begin{tabular}{lccccccc}
\hline Modelamiento & $\chi^{2}(\mathrm{gl})$ & $\chi^{2} / \mathrm{gl}$ & SRMR & CFI & TLI & \multicolumn{2}{c}{ RMSEA $[$ [C 90 \%] } \\
\hline Modelo 1 (Unifactorial) & $448.57(23)$ & 19.50 & .16 & .50 & .54 & .15 & {$[.14, .16]$} \\
Modelo 2 (tres factores oblicuos) & Matriz definida no positiva & & & & & \\
Modelo 3 (dos factores oblicuos) & $\mathbf{4 7 . 3 7 ( 2 3 )}$ & $\mathbf{2 . 0 6}$ & $\mathbf{. 0 5}$ & $\mathbf{. 9 7}$ & $\mathbf{. 9 7}$ & $\mathbf{. 0 4}$ & [.03,.04] \\
Modelo 4 (bifactor) & $43.55(20)$ & 2.18 & .04 & .97 & .97 & .04 & {$[.03, .05]$} \\
\hline
\end{tabular}

Nota $\chi^{2}=$ Chi cuadrado; gl $=$ Grados de libertad; SRMR $=$ Raíz residual estandarizada cuadrática media; RMSEA = Error cuadrático medio de aproximación; CFI = Índice de ajuste comparativo; IC = Intervalos de confianza. La negrita denota el mejor modelo para los datos.

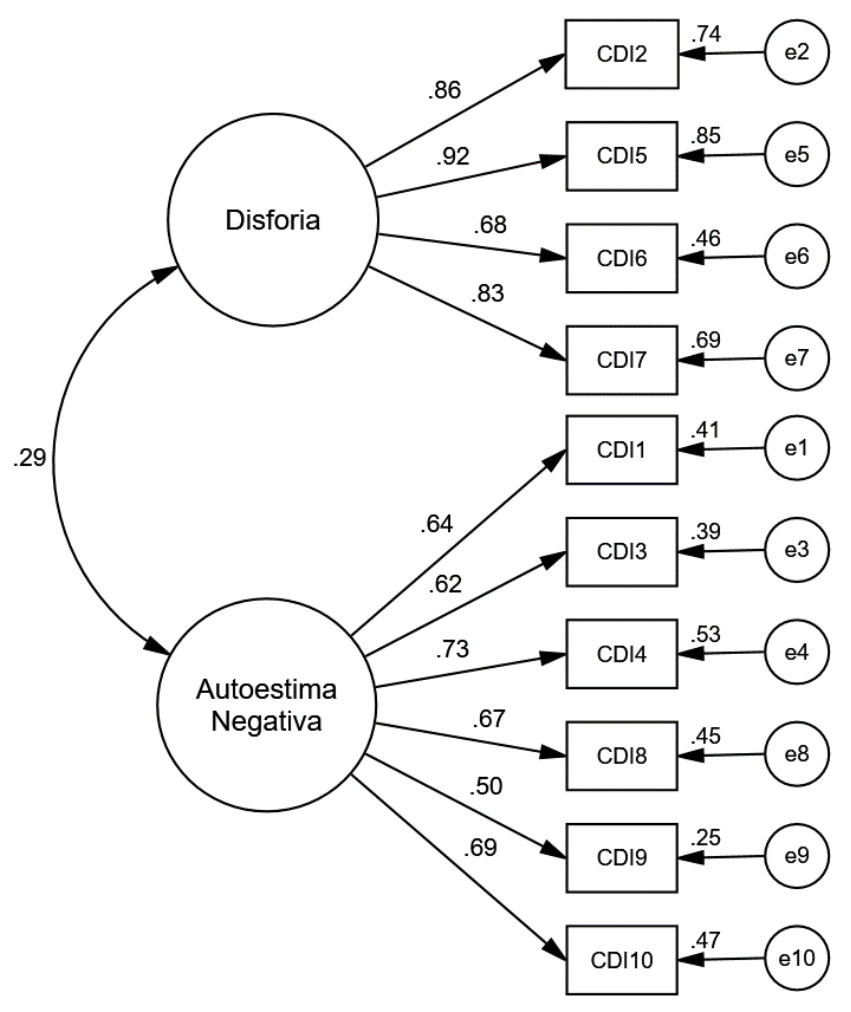

Figura 1. Estructura factorial del CDI (versión peruana)

de las dimensiones Autoestima negativa $(r=.74)$ y Disforia $(r=.62)$, existe una fuerte relación (Cohen, 1988).

\section{Discusión}

La infancia es una de las etapas más importantes en el individuo debido a que las experiencias en estos primeros años son vitales (Costello et al., 2011). La identificación temprana de un niño con depresión reduce la aparición de efectos nocivos en la calidad de vida y el estado de ánimo del infante (Acosta-Hernández et al., 2011), manifestados, por ejemplo, en comportamientos de rebeldía en el aula (Del Barrio, 1997). En ese contexto, resulta oportuno validar una medida breve de depre- sión infantil que pueda servir para la detección rápida de síntomas de Disforia y Autoestima negativa. En consecuencia, el objetivo del presente estudio fue proporcionar evidencia sobre la estructura interna, la relación con la versión de 27 ítems y la estimación de la fiabilidad de las puntuaciones del CDI-S (Kovacs, 1992) en una muestra de niños de Lima y Callao (Perú) mediante el análisis factorial, la correlación de Pearson y el coeficiente de consistencia interna omega.

Los resultados indican que el CDI-S tiene una estructura de dos factores que fue comprobada tanto a nivel exploratorio como confirmatorio. Estos hallazgos son diferentes al modelo de tres factores encontrado en niños españoles (Del Barrio et al., 2002) y a los modelos unifactoriales encontrados con muestra de niños suecos (Ahlen y Ghaderi, 2017) y serbios (Stevanovic, 2012). En el caso del estudio español (Del Barrio et al., 2002), la diferencia ha sido suscitada por el empleo del método de estimación Componentes Principales (CP) y la rotación varimax que recientemente ha sido señalado que el $\mathrm{CP}$ no es análisis factorial y que el método de rotación varimax solo se utiliza en presencia de factores poco relacionados entre sí (Ventura-León, 2017), algo que no es advertido por Del Barrio et al. (2002). En el caso del estudio serbio (Stevanovic, 2012), solo se analizó el modelo unifactorial y no otros modelos alternativos. Por otro lado, el estudio sueco (Ahlen y Ghaderi, 2017) concluyó que el CDI-S es mejor interpretado de forma unifactorial a partir del análisis del modelo bifactor basado en el AFE. Por esa razón, en el presente estudio se probó un modelo bifactor en el AFC a pesar de que no existió una fuerte relación entre los factores; en consecuencia, las medidas auxiliares (ECV, $\omega_{h}$ y $\omega_{h s}$ ) sugirieron el descarte de un factor general, por presentar una varianza común de $35 \%$ y al observar que los factores específicos presentaban fiabilidades superiores a las del factor general.

Finalmente, los resultados coinciden con el estudio en niños colombianos (Yánez et al., 2017) aunque conceptualmente son diferentes porque en dicho estudio los 
dos factores fueron denominados como depresión y ansiedad. Pese a ello, en el presente estudio, que también se reporta una estructura con dos factores, se prefiere la denominación disforia y Autoestima negativa por guardar concordancia teórica con la versión original (Kovacs, 1992) y por ser síntomas principales de la depresión infantil desde hace mucho tiempo (Weinberg et al., 1973); manteniéndose vigente en la comprensión de la depresión infantil (Borges et al., 2016) y en un último estudio del CDI-S (Ahlen y Ghaderi, 2017).

El hecho de modificar el ítem 7 fue una decisión en aras de mejorar la comprensión del ítem en la población infantil peruana ya que es conocido que este tipo de dificultades puede ocasionar sesgos lingüísticos y afectar las puntuaciones de la prueba (ITC, 2018). De esta forma los niños comprendían el sentido semántico del enunciado. Por otro lado, el ítem 1 («Estoy triste...») se organiza en el factor I (Autoestima negativa) porque es el estado de ánimo en que se encuentra el niño cuando siente rechazo consigo mismo, soledad, indefensión y pesimismo.

Por otro lado, la fiabilidad fue estimada mediante el coeficiente $\omega$ y no a través del alfa de Cronbach debido a la utilización de un modelo factorial y a la observación de que las cargas factoriales no son equivalentes, incumpliendo así el supuesto de tau-equivalencia (Cho y Kim, 2015). El modelo bidimensional presentó un $\omega$ por encima del .70 recomendado (Ventura-León y Caycho-Rodríguez, 2017). Estos hallazgos son ligeramente superiores a los de estudios previos (Ahlen y Ghaderi, 2017; Del Barrio et al., 2002; Stevanovic, 2012), aunque esto puede deberse al cambio en el coeficiente, aún reflejan una buena proporción de varianza común entre los ítems.

A pesar de los resultados obtenidos, existen ciertas limitaciones en el estudio. En primer lugar, la población es no-clínica y al ser una prueba que mide una psicopatología es necesario verificar la estructura interna en la población clínica. En segundo lugar, la cantidad de participantes para examinar la correlación entre la versión breve y extensa puede considerarse pequeña por lo que se recomienda ampliar el número de participantes. En tercer lugar, sería útil aplicar el CDI-S en dos momentos temporales diferentes para verificar la estabilidad de las puntuaciones del instrumento a modo de un test-retest (Cho y Kim, 2015). En cuarto lugar, sería bueno examinar la correlación del CDI-S con otro instrumento que midiera teóricamente un constructo relacionado con la depresión con la finalidad de contar con información del funcionamiento predictivo del CDI-S.

Se concluye que los resultados encontrados en el presente estudio sugieren que el CDI-S debe ser interpretado como una medida bidimensional que examina dos síntomas principales de la depresión infantil como son la Disforia y el Autoestima negativa y cuyo comportamiento es similar a la versión extensa en niños escolares peruanos sin diagnóstico clínico, situación que resulta de interés para futuros estudios en el ámbito de la psicología infantil.

\section{Conflictos de intereses}

Los autores declaran que no existen conflictos de intereses.

\section{Referencias}

Acosta-Hernández, M. E., Mancilla-Percino, T., Correa-Basurto, J., Saavedra-Vélez, M., Ramos-Morales, F. R., Cruz-Sánchez, J. S., y Niconoff, S. D. (2011). Depresión en la infancia y adolescencia: enfermedad de nuestro tiempo. Archivos de Neurociencias, 16(1), 20-25.

Ahlen, J., \& Ghaderi, A. (2017). Evaluation of the Children's Depression Inventory-Short Version (CDI-S). Psychological Assessment, 29(9), 1157-1166. https://doi.org/10.1037/ pas0000419

Alcázar, A. I. R., Méndez Carrillo, F. J., Olivares, J., Montoya Fernández, M., Espada Sánchez, J. P., y Sánchez Meca, J. (2002). Tratamiento psicológico de la depresión infantil y adolescente: ¿evidencia o promesa? - Dialnet. Revista de Psicología Clínica Con Niños y Adolescentes, 10(3), 563-580.

Allgaier, A.-K., Frühe, B., Pietsch, K., Saravo, B., Baethmann, M., \& Schulte-Körne, G. (2012). Is the Children's Depression Inventory Short version a valid screening tool in pediatric care? A comparison to its full-length version. Journal of Psychosomatic Research, 73(5), 369-374. https://doi.org/10.1016/j.jpsychores. 2012.08.016

Bernaras-Iturrioz, E., Jauregizar- Albonigamayor, J., SoroaUdabe, M., Ibabe-Erostarbe, I., y de las Cueva-Hevia, C. (2013). Evaluación de la sintomatología depresiva en el contexto escolar y variables asociadas. Anales de Psicología, 29(1), 131-140. https://doi.org/10.6018/analesps.29.1.137831

Borges, L., y Angeli Dos Santos, A. (2016). Sintomatología depresiva y desempeño escolar: un estudio con niños brasileños. Ciencias Psicológicas, 10(2), 189-197.

Borges, L., Baptista, M. N., \& De Oliveira Serpa, A. L. (2016). Structural analysis of depression indicators scale-children and adolescents (BAID-IJ): A bifactor-ESEM approach. Temas Em Psicologia, 25(2), 545-552. https://doi.org/10.9788/tp2017.2-08

Camuñas, N., Mavrou, I., Y Miguel-Tobal, J. J. (2019). Ansiedad y tristeza-depresión: Una aproximación desde la teoría de la indefensión-desesperanza. Revista de Psicopatología y Psicología Clínica, 24(1), 19-28. https://doi.org/10.5944/ rppc. 23003

Caqueo-Urízar, A., Urzúa, A., \& de Munter, K. (2014). Mental health of indigenous school children in Northern Chile. BMC Psychiatry, 14(1), 1-7. https://doi.org/10.1186/1471-244X-14-11

Cho, E., \& Kim, S. (2015). Cronbach's coefficient alpha: Well-known but poorly understood. Organizational Research Methods, 18(2), 207-230. https://doi.org/10.1177/1094428114555994 
Cohen, J. (1988). Statistical power analysis for the behavioral sciences. Abingdon, UK: Routledge.

Costello, E. J., Copeland, W., \& Angold, A. (2011). Trends in psychopathology across the adolescent years: What changes when children become adolescents, and when adolescents become adults? Journal of Child Psychology and Psychiatry, 52(10), 1015-1025. https://doi.org/10.1111/j.1469-7610.2011.02446.x

De la Vega, R., Racine, M., Sánchez-Rodríguez, E., Solé, E., Castarlenas, E., Jensen, M. P., ... Miró, J. (2016). Psychometric properties of the short form of the Children's Depression Inventory (CDI-S) in young people with physical disabilities. Journal of Psychosomatic Research, 90, 57-61. https://doi. org/10.1016/j.jpsychores.2016.09.007

Del Barrio V. (1997). Depresión infantil: causas, evaluación y tratamiento. Barcelona, España: Ariel.

Del Barrio V, Capilla M, Olmedo M, y Colodrón F. (2002). Primera adaptación del CDI-S a población española. Acción Psicológica, 1(3), 263-272. https://doi.org/10.5944/ ap.1.3.558

Delmas, M.-C., Guignon, N., Chan Chee, C., Fuhrman, C., Herbet, J.-B., \& Gonzalez, L. (2011). Asthma and Major Depressive Episode in Adolescents in France. Journal of Asthma, 48(6), 640-646. https://doi.org/10.3109/02770903.2011.585410

Demir, T., Karacetin, G., Demir, D. E., \& Uysal, O. (2011). Epidemiology of depression in an urban population of Turkish children and adolescents. Journal of Affective Disorders, 134(1-3), 168-176. https://doi.org/10.1016/j.jad.2011.05.041

Freiberg Hoffmann, A., Stover, J. B., de la Iglesia, G., \& FernándezLiporace, M. (2013). Correlaciones policóricas y tetracóricas en estudios factoriales exploratorios y confirmatorios. Ciencias Psicológicas, 7(2), 151-164.

Hu, L., \& Bentler, P. M. (1999). Cutoff criteria for fit indexes in covariance structure analysis: Conventional criteria versus new alternatives. Structural Equation Modeling: A Multidisciplinary Journal, 6(1),1-55.https://doi.org/10.1080/10705519909540118

Instituto Nacional de Salud Mental Honorio Delgado - Hideyo Noguchi. (2018). Estudio epidemiológico de salud mental de niños y adolescentes en Lima Metropolitana y Callao. Anales de Salud Mental, 38(Supl.1), 1-219.

ITC Guidelines for Translating and Adapting Tests (Second Edition). (2018). International Journal of Testing, 18(2), 101134. https://doi.org/10.1080/15305058.2017.1398166

Ivarsson, T., Svalander, P., \& Litlere, O. (2006). The Children's Depression Inventory (CDI) as measure of depression in Swedish adolescents. A normative study. Nordic Journal of Psychiatry, 60(3), 220-226. https://doi.org/10.1080/08039480600636395

James, S. L., Abate, D., Abate, K. H., Abay, S. M., Abbafati, C., Abbasi, N., ... Abdelalim, A. (2018). Global, regional, and national incidence, prevalence, and years lived with disability for 354 diseases and injuries for 195 countries and territories, 1990-2017: a systematic analysis for the Global Burden of Disease Study 2017. The Lancet, 392(10159), 1789-1858. https://doi.org/10.1016/s0140-6736(18)32279-7

Jaureguizar, J., Bernaras, E., \& Garaigordobil, M. (2017). Child Depression: Prevalence and Comparison between SelfReports and Teacher Reports. The Spanish Journal of Psychology, 20(1), 1-20. https://doi.org/10.1017/sjp.2017.14

Kovacs, M. (1992). Children's Depression Inventory. North Tonawanda, USA: Multi-Health Systems.
Lara-Ros, M. R., Rodríguez-Jiménez, T., Martínez-González, A. E., y Piqueras, J. A. (2017). Relación entre el bullying y el estado emocional y social en niños de educación primaria. Revista de Psicología Clínica con Niños y Adolescentes, 4(1), 59-64.

Lorenzo-Seva, U., \& Ferrando, P. J. (2006). FACTOR: A computer program to fit the exploratory factor analysis model. Behavior Research Methods, 38(1), 88-91.https://doi.org/10.3758/bf03192753

Martinez, A., y Rosselló, J. (2015). Depresión y funcionamiento familiar en niños/as y adolescentes puertorriqueños/as. Revista Puertorriqueña de Psicología, 10(1), 215-245.

McLeod, B. D., Weisz, J. R., \& Wood, J. J. (2007). Examining the association between parenting and childhood depression: A meta-analysis. Clinical Psychology Review, 27(8), 986-1003. https://doi.org/10.1016/j.cpr.2007.03.001

Meehan, F., Houghton, F., Cowley, H., Houghton, S., \& Kelleher, K. (2008). Children's depression, gender and age norms for an Irish national (primary) school population. Irish Journal of Psychological Medicine, 25(3), 88-94. https://doi.org/10.1017/ s0790966700011071

Mueller, R. O., \& Hancock, G. R. (2008). Best practices in structural equation modeling. In J.W. Osborne (Ed.), Best practices in quantitative methods (Chapter 32, pp. 488-508). London, UK: Sage

Osborne, J. W., Costello, A. B., \& Kellow, J. T. (2008). Best practices in exploratory factor analysis. Best Practices in Quantitative Methods, 86-99. https://doi.org/10.4135/9781412995627.d8

Quintana, L., y Maldonado, E. (2016). Sintomatología de depresión en niños y niñas que han sido testigos de violencia en la calle que viven en ciudad Juárez. Revista Electrónica de Psicología Iztacala, 19(3), 1014-1026.

Reise, S. P., Scheines, R., Widaman, K. F., \& Haviland, M. G. (2013). Multidimensionality and structural coefficient bias in structural equation modeling: A bifactor perspective. Educational and Psychological Measurement, 73(1), 5-26. https://doi.org/10.1177/0013164412449831

Rosseel, Y. (2012). Lavaan: An R package for structural equation modeling and more. Version 0.5-12 (BETA). Journal of statistical software, 48(2), 1-36.

Smits, I. A. M., Timmerman, M. E., Barelds, D. P. H., \& Meijer, R. R. (2014). The Dutch symptom checklist-90-revised. European Journal of Psychological Assessment. 31(1), 1-9. https://doi. org/10.1027/1015-5759/a000233

Stevanovic, D. (2012). Childhood depression and anxiety disorders in Serbia: a psychometric study of four screening questionnaires. Epidemiology and Psychiatric Sciences, 21(1), 111-116. https://doi.org/10.1017/S2045796011000655

Sun, S., \& Wang, S. (2015). The Children's Depression Inventory in Worldwide Child Development Research: A Reliability Generalization Study. Journal of Child and Family Studies, 24(8), 2352-2363. https://doi.org/10.1007/s10826-014-0038-x

Timmerman, M. E., \& Lorenzo-Seva, U. (2011). Dimensionality assessment of ordered polytomous items with parallel analysis. Psychological Methods, 16(2), 209. https://doi. org/10.1037/a0023353

Ventura-León, J. (2017). ¿Componentes principales o factores comunes?: Comentarios al artículo de Hederich-Martínez y Caballero-Domínguez (2016). CES Psicología, 10(1), 146-147.

Ventura-León, J., y Caycho-Rodríguez, T. (2017). El coeficiente Omega: un método alternativo para la estimación de la 
confiabilidad. Revista Latinoamericana de Ciencias Sociales, Niñez y Juventud, 15(1), 625-627.

Weinberg, W. A., Rutman, J., Sullivan, L., Penick, E. C., \& Dietz, S. G. (1973). Depression in children referred to an educational diagnostic center: Diagnosis and treatment: Preliminary report. The Journal of Pediatrics, 83(6), 1065-1072. https:// doi.org/10.1016/s0022-3476(73)80552-9
Yánez Botello, C., Rozo Sánchez, M., Avendaño, B., Barraza, L., Gutiérrez, D., y Sánchez Silva, N. (2017). Análisis psicométrico del Children's Depression Inventory-Short y porcentaje de niños y niñas colombianos con síntomas depresivos. Cultura Educación y Sociedad, 8(1), 25-42. https://doi.org/10.17981/ cultedusoc.8.1.2017.02 
